\title{
Thermodynamic modelling of hydrogen in Nominally Anhydrous Minerals (NAMs): current strategies
}

\section{JOSÉ ALBERTO PADRÓN-NAVARTA}

Instituto Andaluz de Ciencias de la Tierra, CSIC-Universidad de Granada

Presenting Author: alberto.padron@csic.es

Much progress has been made in recent years on the mechanisms and physical consequences of hydrogen incorporation in minerals that they do not contain $\mathrm{H}_{2} \mathrm{O}$ as a major constituent in their structure (aka. Nominally Anhydrous Minerals, NAMs). Despite the amount of new experimental data [1], the extrapolation to regions outside the experimental range (typically at low subsolidus temperature and waterundersaturated conditions) relies on models that do not capture the complexities observed in nature. A remarkable exception is the only model that is able to handle water-undersaturated conditions (pHMELTS [2]) by using an iterative algorithm that converge the thermodynamically modelled $\mathrm{H}_{2} \mathrm{O}$ chemical potential and the water content in the NAMs fitted from fluid saturated experiments to maintain mass balance constrains. Yet simple, these models track first order consequences for the adiabatic near-fractional decompression melting [2]. Although powerful such model is not able to account for the importance of several hydrated point defects with different dependencies on water activity [3], the role of trace element in NAMs in controlling low water concentrations and importantly how the stability of other phases (such as amphiboles) will impact on both, water activity and trace element partitioning.

One approach, presented here, is to develop a thermodynamic treatment of intrinsically and extrinsically (i.e. trace elements) hydrated point defects that can be combined with robust freeenergy minimizations strategies (such as Perple_X [4]). This will allow to track changes in bulk water contents and changes in mineral assemblages at subsolidus and supersolidus conditions. Hydrogen incorporation is modeled as part of a solid solution between a fictive hydrous and a real anhydrous end-members (e.g. $\mathrm{Mg}_{2} \mathrm{H}_{4} \mathrm{O}_{4}$ and $\mathrm{Mg}_{2} \mathrm{SiO}_{4}$ respectively for forsterite with hydrated $\mathrm{Si}$-vacancies) having regular solution properties in the Henry's law limit. The free energy of the fictive hydrous endmember in the anhydrous structure is found from known endmember thermodynamic properties and modified by a DQF parameter (Darken's quadratic formalism) based on calibrated experiments for the particular hydrous point defect involved.

[1] Padrón-Navarta \& Hermann, JGR 122 (2017). [2] Asimow et al. G3 5 (2004). [3] Tollan, et al. Prog. Earth Planet. Sci. 4 (2017). [4] Connolly G3 10 (2009). 